DOI: https://doi.org/10.46296/yc.v5i8edespmar.0099

\title{
NUEVOS MERCADOS DE ECONOMÍA POPULAR Y SOLIDARIA. UN ENFOQUE ESTRATÉGICO DESDE EL EMPRENDIMIENTO LOCAL
}

\section{NEW MARKETS OF POPULAR AND SOLIDARITY ECONOMY. A STRATEGIC APPROACH FROM LOCAL ENTREPRENEURSHIP}

\author{
López-Lucas Luis Eduardo ${ }^{1 *}$; Hidalgo-Ávila Argelio Antonio ${ }^{2}$ \\ ${ }^{1}$ Ingeniero Agroindustrial, Universidad Laica Eloy Alfaro de Manabí. Manta, Ecuador. Correo: \\ llopez9023@pucesm.edu.ec. ORCID ID: https://orcid.org/0000-0001-6915-5818 \\ ${ }^{2}$ Docente del Área de Posgrado en la Pontificia Universidad Católica del Ecuador, sede \\ Manabí. Portoviejo, Ecuador. Docente Facultad de Contabilidad y Auditoría, Universidad Laica \\ Eloy Alfaro de Manabí. Manta, Ecuador. Correo: ahidalgo@pucesm.edu.ec. ORCID ID: \\ https://orcid.org/0000-0002-1952-5312
}

\begin{abstract}
Resumen
La investigación tiene como objetivo principal contextualizar las iniciativas de emprendimiento que surgen desde los mercados de economía popular y solidaria; el enfoque es de carácter cualitativo e inductivo, con un nivel descriptivo analítico, documental y de campo, para lo cual se tomó como unidad de estudio la Coordinación de Desarrollo Económico del Gobierno Autónomo Descentralizado del (GAD) Manta, en la localidad del cantón Manta, provincia de Manabí (Ecuador), en la que participó como informante directa su responsable, así como el también conocer la postura de investigadores relacionados con el tema, todos mediante una entrevista en profundidad. La técnicas de análisis de los resultados se basó en las propuestas dadas desde la Teoría Fundamentada en cuanto a su saturación teoría, triangulación, comprensión analítica y proceso de interpretación; entre los resultados relevantes se encuentra la posibilidad de establecer un enfoque de manera estratégica, en la que se incluyan variables como sostenibilidad, innovación y colaboración, las cuales deben ser consideradas al momento de establecer estrategias de emprendimiento en mercados de economía popular y solidaria.
\end{abstract}

Palabras claves: Mercado de economía popular y solidaria, emprendimiento, estrategias.

\begin{abstract}
The main objective of the research is to contextualize the entrepreneurship initiatives that arise from the popular and solidarity economy markets; The approach is qualitative and inductive, with a descriptive, analytical, documentary and field level, for which the Economic Development Coordination of the Decentralized Autonomous Government of (GAD) Manta was taken as a study unit, in the town of Manta canton., province of Manabí (Ecuador), in which the person in charge participated as a direct informant, as well as knowing the position of researchers related to the subject, all through an in-depth interview. The analysis techniques of the results were based on the proposals given from the Grounded Theory regarding its saturation theory, triangulation, analytical understanding and interpretation process; Among the relevant results is the possibility of establishing a strategic approach, in which variables such as sustainability, innovation and collaboration are included, which must be considered when establishing entrepreneurship strategies in popular and solidarity economy markets.
\end{abstract}

Keywords: Popular and solidarity economy market, entrepreneurship, strategies.

Información del manuscrito:

Fecha de recepción: 18 de enero de 2021.

Fecha de aceptación: 19 de marzo de 2021.

Fecha de publicación: 29 de marzo de 2021 


\section{Introducción}

El surgimiento de nuevos mercados trae consigo mismo la incorporación de novedosas situaciones que bien requieren estructurar estrategias innovadoras para su abordaje; el mercado en sí mismo se entiende como un espacio de intercambio, en las que actores comerciales negocian sobre el valor de un producto o servicio, estableciéndose este proceso de intercambio a través de reglas previamente acordadas.

Desde el seno de la presente investigación se entiende que, los nuevos mercados, nacen producto de ciertas y determinadas necesidades en la que la población requirente de productos y servicios en la que está dispuesta a hacer uso, pero en condiciones diferentes a los mercados tradicionales ya establecidos. Por ello, se hace necesario reglas ajustadas al mercado naciente, y por ende estrategias con enfoque estratégico empresarial diferente al tradicional para su abordaje. La investigación centra su interés en los mercados que surgen desde las perspectivas de la economía popular y solidaria, para lo cual se hace indispensable una delimitación basada en lo estratégico que bien permite dar cumplimiento a la línea de investigación en la que se enmarca este trabajo como lo es el de Responsabilidad Social, Economía Popular y Solidaria y Gobernanza, con enfoque de emprendimiento estratégico.

Un mercado basado en una estructura conceptual en la que se le denomine de economía popular y solidaria requiere para su estudio una clara delimitación sobre los aspectos que la diferencian, a fin de lograr una sólida significación en su concepción. Para ello, Unceta (2014) aclara que un mercado nace "para poder generar integración y asociación entre las partes, el intercambio precisa de un sistema que se rige sobre la base de precios $y$ de mecanismos de oferta $y$ demanda" (p.4). Estos mercados se manifiestan por el bajo ingreso que tienen los habitantes que viven en zonas no privilegiadas 0 residenciales campesinas y que requieren, en muchas ocasiones, de la tutela de las autoridades gubernamentales para su organización y demarcación; 
además de una característica muy peculiar: son poblaciones desatendidas por parte de la misma autoridad gubernamental en términos de servicios básicos, y de los generadores de productos y servicios; estos últimos consideran que este tipo de población vulnerable no tiene los recursos económicos para atender una oferta en los mercados tradicionales.

Por otro lado, a nivel demográfico, los mercados emergentes con la etiqueta de economía popular y solidaria tienden en su mayoría a resaltar por una dominante población joven y con unas ratios de natalidad muy elevados, lo que indica que la población irá en acelerado aumento. Uno de los factores determinantes de esta situación de crecimiento acelerado es por la ausencia de una educación formal, familiar, y hasta financiera en los círculos neurálgicos de la población en condición vulnerable, en especial a los más jóvenes que se encuentran en este segmento. En este sentido, se hace indispensable que el sector gubernamental y empresarial diseñen estrategias en las que se permitan ofrecer de manera accesible y, a su vez rentable, productos adaptados a este contexto y sus necesidades. La mayor parte de las necesidades está focalizada en los productos alimenticios, y más aún en los provenientes del sector agrícola.

Lógicamente, las iniciativas de emprendimiento ameritan una serie de condicionamientos que no solo dependen de una estrategia gubernamental y del apoyo del sector empresarial, sino que el propio habitante este educado sobre la importancia de la propia iniciativa y su significado para la vida diaria. Países como Ecuador, con altos índices de iniciativas en emprendimiento, no son ajenos a la situación global latinoamericana en los términos de apalancamiento de mercados de economía popular y solidaria; en el país existen leyes y normas que impulsan el desarrollo de estos mercados, observándose que la estructura gubernamental, en casi todos sus niveles, establece acciones promotoras para concientizar a la población más vulnerable sobre esta alternativa que bien les permita redimensionarse hacia una condición de ciudadano productivo, 
lógicamente dentro del concepto de una economía popular.

La economía popular, según Castro (2018) se define "como un conjunto inorgánico de actividades realizadas por trabajadores, subordinadas directa o indirectamente a la lógica del capital, que deben accionarse para que se transforme en un subsistema económico orgánicamente articulado, centrado en el trabajo" (p.38); es decir, la acumulación de capital no es la razón principal de estos mercado cuya intención se focaliza en dar ocupación laboral a una población que busca un sustento que les permita ayudar a la familia el poder cubrir sus necesidades básicas; en la que se busca el aprovechamiento de las condiciones que brinda su propio entorno, como lo son los casos de la tierra y su uso para la agricultura; el arte urbano y local expresado a través de la orfebrería y la artesanía, entre otras iniciativas de características similares. Una de las estrategias gubernamentales más utilizadas en el Ecuador, con una visión local, es la incorporación de programas de economía popular y solidaria en los planes de acción de cada cantón, cuyos proyectos se orientan al buen uso, además de equilibrado, de este tipo de mercado, encontrándose en el mismo propuestas muy atractivas.

El propósito de la investigación está en visibilizar el contexto actual en la que se demarca el mercado de economía popular y solidaria en el Ecuador, específicamente en el cantón Manta (provincia de Manabí), como eje de campo de la investigación; desde la experiencia en la temática, los investigadores consideran importante analizar las estrategias que han sido aplicadas por el Gobierno Autónomo Descentralizado Manta (GADManta) mediante el impulso de programas como Manta Emprendedora y Manta Innovadora. Estos programas forman parte de una agenda de contacto entre el GAD-Manta y los beneficiarios, en las que estos últimos reciben un apoyo institucional para el fortalecimiento de sus iniciativas; sin embargo, los resultados aún están por verse en cuanto a la sostenibilidad de dichas iniciativas.

Ahora bien, tomando en consideración los aspectos mencionados anteriormente, desde la investigación surge la siguiente 
interrogante: ¿Qué enfoque estratégico deben considerar los emprendimientos para una incursión exitosa en mercados de economía popular y solidaria? A fin de dar respuesta a esta interrogante, se plantean los siguientes objetivos: Analizar las estrategias de emprendimiento que surgen desde los mercados de economía popular y solidaria, así como el identificar las iniciativas de emprendimiento que permiten contribuir con los nuevos mercados de economía popular y solidaria; además el describir las condiciones que se requieren para que las iniciativas de emprendimiento resulten de manera exitosa.

\section{Desarrollo}

\section{1.- Economía popular y} solidaria. Una mirada desde el emprendimiento

Existen múltiples acepciones sobre el tema de la economía popular y solidaria, lo cual se interpreta según el contextos y posturas sociopolíticas del referente; sin embargo, la investigación procura establecer una línea conceptual autónoma que contribuya develar realidades presentes $\mathrm{y}$, por ende, generar una alternativa viable basada en el discurso del emprendimiento productivo.

En el Ecuador, su Constitución de la República establece como se constituye su economía y, de manera diferenciada lo referente a la economía popular y solidaria en su artículo 283:

El sistema económico se integrará por las formas de organización económica pública, privada, mixta, popular $y$ solidaria, y las demás que la Constitución determine. La economía popular y solidaria se regulará de acuerdo con la ley e incluirá a los sectores cooperativistas, asociativos $y$ comunitarios

(Asamblea

Constituyente, 2008)

Agregando en su artículo 288 "Se priorizarán los productos y servicios nacionales, en particular los provenientes de la economía popular y solidaria, y de las micro, pequeñas $y$ medianas unidades productivas" (2008). Ambos artículos direccionan cual debe ser el comportamiento de las estructuras gubernamentales frente 
al sistema económico, específicamente en cuanto a la forma de economía popular y solidaria.

Por su parte, el Plan de Desarrollo Toda Una Vida 2017-2021 (p.77), en su eje 2 denominado: Economía al Servicio de la Sociedad, establece los objetivos en las cuales se sustenta la incorporación de la economía popular y solidaria en la vida cotidiana del ciudadano, destacándose: Objetivo 4: Consolidar la sostenibilidad del sistema económico social y solidario, y afianzar la dolarización; objetivo 5: Impulsar la productividad y competitividad para el crecimiento económico sostenible de manera redistributiva y solidaria; y el objetivo 6: Desarrollar las capacidades productivas y del entorno para lograr la soberanía alimentaria y el Buen Vivir rural. A través de estos objetivos, plasmados en el plan de desarrollo vigente, se busca que las entidades e instituciones gubernamentales prioricen el garantizar condiciones para que la ciudadanía ejerza el compromiso establecido en la carta magna, viabilizando oportunidades para el que el propio ciudadano desarrolle alternativas viables para el sustento propio y familiar, a través del trabajo productivo.

Ahora bien, con la idea de establecer un acercamiento desde una postura socioeconómica, en la que Cotera (2008) refiere que la economía popular solidaria "se consolida con base en el apoyo mutuo, la cooperación y el intercambio de conocimientos, mercados o tecnología" (p.42), agregando que la estrategia debe de ser "el maximizar el factor solidario para lograr la satisfacción de las necesidades básicas y generar acumulativamente procesos de desarrollo desde lo local, donde emerjan unidades productivas" (p.43); estas unidades productivas son las que podemos definir como iniciativas de emprendimiento. Para Vegas (2017) el emprendimiento "en sí mismo tiene varios matices, los cuales se diferencian según el contexto de aplicación y la capacidad de respuesta que se tiene frente al mercado" (p.7), lo cual refuerza un poco a lo expresado por Cotera (2008) en la que considera que las iniciativas de emprendimiento en el contexto de la economía popular y solidaria son "el 
espacio de un mercado más solidario que competitivo, y de un planeamiento del desarrollo de abajo hacia arriba y que tiene en el Estado democratizado su referente más abarcador" (p.43); esto deja claro que el significado de emprendimiento en el contexto del mercado popular y solidario tiene condiciones y expectativas diferentes a las de un emprendimiento que busca abarcar, y hasta controlar, buena parte de aquel tipo de mercado con características propias de la oferta y demanda masiva.

Un emprendimiento debe tener claro cuál es su enfoque estratégico, y sin dudas debe de abarcar diferentes ángulos: composición del mercado en cuanto a usuarios o consumidores posibles, competencia, proveedores, tecnología y, por supuesto, de la cultura innovadora. Lógicamente, la particularidad de cada mercado permite a cada emprendimiento reforzar su direccionamiento empresarial para la conquista de una buena parte del segmento en disputa; muchos le denominan el libre mercado, donde la oferta y la demanda están en constante rivalidad.

Ahora bien, cuando se trata de un mercado cuyas variables están direccionadas sobre la base de la solidaridad, el intercambio equilibrado $y$, por ende, de una asociación de intereses comunes, la idea de un libre mercado se diluye ante los requerimientos exigidos por las instituciones gubernamentales en la que las condiciones para pertenecer a este tipo de mercado son de un orden muy específico. Las instituciones del Estado promueven este tipo de mercado en aras de que sus emprendedores procuren establecer alianzas entre sí y por supuesto con el mismo gobierno a fin de abaratar costos, facilitar el intercambio y de alguna manera, generar oportunidades de negocio para la gran mayoría de los que se insertan en este mercado. Las políticas públicas orientadas para tal fin, así como los programas y proyectos que se proponen, además de la intención de quienes aspiran una oportunidad en este tipo de mercado, siempre estarán presentes. Esta afirmación se da soportada desde el texto constitucional y las normas 0 
resoluciones que emanan para su alcance, y más aún cuando forman parte de los propios planes de desarrollo cantonal; por supuesto, esto último no forma parte del análisis y la discusión investigativa; sin embargo, es importante considerar el reto que representa en sí misma el hecho que se puedan concretar todas las iniciativas de una manera tangible y con el verdadero impacto.

Existen temáticas que han sido debatidas en el seno de la Organización de las Naciones Unidas (ONU) y que han sido plasmadas en varios de sus 17 objetivos del milenio, destacándose el objetivo 12 "Garantizar modalidades de consumo y producción sostenibles", en él se expresa la importancia de elementos y situaciones que se deben de considerar para ser realmente productivos, soportándose desde la estructura social, y más aún en lo familiar; permitiéndose traducir "en una mejor calidad de vida para todos $\mathrm{y}$, además, ayuda a lograr planes generales de desarrollo, que rebajen costos económicos, ambientales y sociales, que aumenten la competitividad y que reduzcan la pobreza" (p.127). Este objetivo 12, presentado y vigente como de desarrollo sostenible por la ONU, hace una invitación directa al uso razonable de las unidades de producción y de sus recursos (naturales / comunes), lo cual redunda en mejores niveles de aceptación por parte de los consumidores, y por ende de sus propios procesos de producción.

\section{Metodología}

La investigación se plantea desde la perspectiva cualitativa, con un nivel descriptivo analítico y de método inductivo, cuyo tipo es documental y de campo, no experimental. La investigación hace uso de la técnica de investigación la observación directa analítica y la entrevista en profundidad. La unidad de estudio en la que se hace el acercamiento investigativo es la Dirección de Desarrollo Productivo, la cual forma parte de la estructura organizativa y funcional del Gobierno Autónomo Descentralizado del cantón Manta, provincia de Manabí. La investigación asume la propuesta dada por la Teoría Fundamentada, planteada por Strauss y Corbin 
(2002) en la que destacan que "esta metodología proporciona un sentido de visión, de adónde quiere ir analista con la investigación" (p.11); la misma resultó de gran utilidad al momento de establecer las estrategias de levantamiento y análisis de la información recabada; así como para su ordenamiento, complementando esta acción con el uso del método hermenéutico para una adecuada comprensión e interpretación de los resultados; con ello se complementa la configuración metodológica propuesta por la investigación.

En cuanto a los instrumentos de recolección de información (observación directa y entrevista), la investigación mantuvo un acercamiento continuo con la unidad de estudio (Dirección de Desarrollo Productivo) para constatar su comportamiento, obteniendo así impresiones muy precisas sobre la dinámica en la que se desenvuelve de manera cotidiana y su vinculación con el tema en estudio. En cuanto a la entrevista en profundidad, la misma se aplicó de manera directa a la Ingeniera Paola Ramon, Coordinadora de Desarrollo
Económico del Gobierno Autónomo Descentralizado del (GAD) cantón Manta; quien con su experiencia y conocimiento sobre la temática permite valorar operativamente los condicionantes que se dan desde el cantón Manta; y además, bajo su coordinación se encuentran los programas Manta Emprendedora y Manta Innovadora; adicionalmente se incorporan los aportes de los investigadores Guillermo Marín Balcazar, PhD (Universidad Tecnológica de Ciudad Juárez, estado de Chihuahua), Luis Alamilla (Instituto Tecnológico de Cd. Juárez (ITCJ), Chihuahua, México) e Hilarión Vegas Meléndez, PhD (Pontificia Universidad Católica del Ecuador), todos reconocidos por su continuo interés y posturas sobre la temática, brindando una visión global, académica y científica a la investigación.

En las entrevistas aplicadas a los actores informantes se observan posturas interesantes y paradigmáticas en las que hay una muestra de la caracterización discursiva propia del ámbito en que se desenvuelven estos actores. Entre los temas abarcados en las entrevistas destacaron las premisas 
que dan sustento a una iniciativa de emprendimiento en mercados de economía popular y solidaria; Ramon (2021) en este punto expresa "Es importante vender el producto o servicio con una historia atrás...[...] tanto es relevante visualizar al comprador el extra por lo que están pagando" (p.1); Marín (2021) indica que en este tipo de emprendimiento "Un indicador importante es el grado de cooperación y beneficios colectivos" (p.2); Alamilla (2021) considera que se puede hacer "a través del desarrollo de formas alternas al lucro total e impersonal de los negocios en una economía de libre mercado" (p.3); por su parte Vegas (2021) manifiesta que en "la mayoría de las ocasiones esta se da por una necesidad de generar ingresos de manera inmediata, sin medir las condiciones que requieren para su éxito y sostenibilidad; además, sus parámetros de funcionamiento están regulados por autoridades locales" (p.4).

En cuanto a las estrategias innovadoras vinculadas al emprendimiento cuya orientación estén dadas a una significativa potenciación en los mercados de economía popular y solidaria; Ramon (2021) recomienda que es necesario "pensar las nuevas formas de hacer comercio [...] Esto además son mercados en los que podemos llegar al consumidor de manera directa, sin intermediación" (p.1); el investigador Marín (2021) considera manifiesta que "tanto el desarrollo local como la economía solidaria coinciden en el objetivo de contribuir a mejorar la calidad de vida de los miembros de una comunidad, lo cual se basa en iniciativas locales, a través de la transformación estructural" (p.2); Alamilla (2021) en ese mismo orden, se expresa que "esa misma inercia del mercado tradicional las empujará a competir por espacios en los mercados lucrativos, pero con una eficiente capacitación y concientización de la importancia de mejorar mediante la creatividad e innovación" (p.3); por su parte Vegas (2021) refiere que "todo enfoque estratégico debe estar acompañado de un contenido innovador y diferenciador, ahora bien, este tipo de mercado requiere para su potenciación, que sus usuarios tengan confianza en la calidad del producto y un solidario 
acompañamiento en cuanto al precio" (p.4).

Entre las condiciones que debe tener un emprendimiento en el mercado de economía popular y solidaria para que realmente sea productivo en lo local, Ramon (2021) advierte que el mercado "debe tener calidad, es decir cumplir con los requisitos que exige el consumidor, estar adecuado a la normativa nacional, mantener un sistema de calidad básico, y cumplir con los principios de la EPS" (p.1); Marín (2021) destaca que "la primera condición es el aprovechamiento de recursos endémicos propios de la zona, Buscar explotar oportunidades de negocios externos viendo las oportunidades internas" (p.2); Alamilla (2021) expone que "no por ser un mercado de economía popular al cual servirán, los productos serán de baja calidad, se trata de que sobrevivan en el tiempo, sean sustentables, y extiendan sus operaciones en escala de ser posible" (p.3); a lo que Vegas (2021), por su parte resalta "la intervención de una política pública local en este tipo de mercado siempre busca un sentido colaborativo entre sus actores, focalizándose en una productividad con responsabilidad colectiva, en producir y consumir solo lo necesario; afianzando la cultura del ahorro" (p.4)

Sobre el enfoque estratégico que deben considerar los emprendimientos para una incursión exitosa en mercados de economía popular y solidaria; Ramon (2021) considera que es necesario "primero tener un plan de negocios consolidado, que incluya un estudio de mercado, canales de comercialización, plan financiero y de financiamiento" (p.1); Marín (2021) cree que entre los grandes retos "es enfrentar los problemas que se presentan en la actualidad, como son la pobreza, la inequidad y el daño al medioambiente, que contribuya a equiparar el desarrollo social con el desarrollo tecnológico y productivo" (p.2); Alamilla (2021) indica la importancia en "el cambio de paradigma, del reparto de la ganancia o las utilidades debería ser como un medio para vivir, no un fin, algo sumamente complicado de lograr [...] cumplir objetivos impensables, a los nuevos empresarios sociales con la 
educación y capacitación" (p.3); en este contexto Vegas (2021) expresa que "todo emprendimiento exitoso, basa sus estrategias en el conocimiento, la educación que brindas al consumidor y la capacidad de ser innovador, sin importar el tipo de mercado en sí" (p.4).

\section{Resultados}

Siguiendo los pasos que proponen Strauss y Corbin (2002) en la fase de "descripciones $u$ ordenamiento conceptuales (clasificar o elaborar)" (p.32), la investigación asumió un comportamiento analítico para la construcción de la ruta de análisis, partiendo dese la saturación teórica hasta llegar a la ordenación de las teorías vinculantes con la investigación haciendo uso de la comparación; en este sentido, siguiendo lo propuesto por Vegas (2016) la comparación teórica "genera un marco conceptual mínimo que bien permite a los investigadores estimular el uso del pensamiento acerca de las propiedades y dimensiones para la construcción de las categorías, lo cual ayuda en la interpretación establecer una epistemología de lo encontrado" (p.17); esta categorías, luego de un proceso de análisis hermenéutico (comprensión e interpretación), dieron origen a los descriptores que se muestran como aporte de la investigación.

Tal como se observa en la tabla 1 , se presentan las categorías y descriptores resultantes, producto de la triangulación entre las teorías revisadas, la postura de los actores entrevistados y el análisis comparativo desarrollado por parte de los investigadores. Veamos:

Tabla 1. Categorías y Descriptores Emergentes de la Entrevista en Profundidad.

\begin{tabular}{|c|c|c|c|}
\hline Entrevistado & Códigos en vivo & Categorías & Descriptores \\
\hline $\begin{array}{l}\text { AC: Paola Ramón } \\
\text { (2021) }\end{array}$ & $\begin{array}{c}\text {-Visión de mercados } \\
\text { "históricos / } \\
\text { artesanales" } \\
\text {-Negocio "cara a } \\
\text { cara" } \\
\text {-Calidad y normas } \\
\text {-Plan de negocio }\end{array}$ & $\begin{array}{l}\text {-Mercados de } \\
\text { Saberes } \\
\text {-Compromiso } \\
\text {-Sustentabilidad }\end{array}$ & $\begin{array}{c}\text { - Mercado } \\
\text { diferenciado y } \\
\text { sustentable } \\
\text {-Modelo autónomo } \\
\text { de gestión }\end{array}$ \\
\hline $\begin{array}{l}\text { EAl: Marín B. } \\
\text { Guillermo (2021) }\end{array}$ & $\begin{array}{c}\text {-Grado de } \\
\text { cooperación y } \\
\text { beneficios } \\
\text {-Transformación } \\
\text { estructural }\end{array}$ & $\begin{array}{l}\text {-Sustentos del buen } \\
\text { vivir local }\end{array}$ & $\begin{array}{l}\text {-Emprendimiento } \\
\text { pensado en la } \\
\text { sostenibilidad del } \\
\text { mercado }\end{array}$ \\
\hline
\end{tabular}




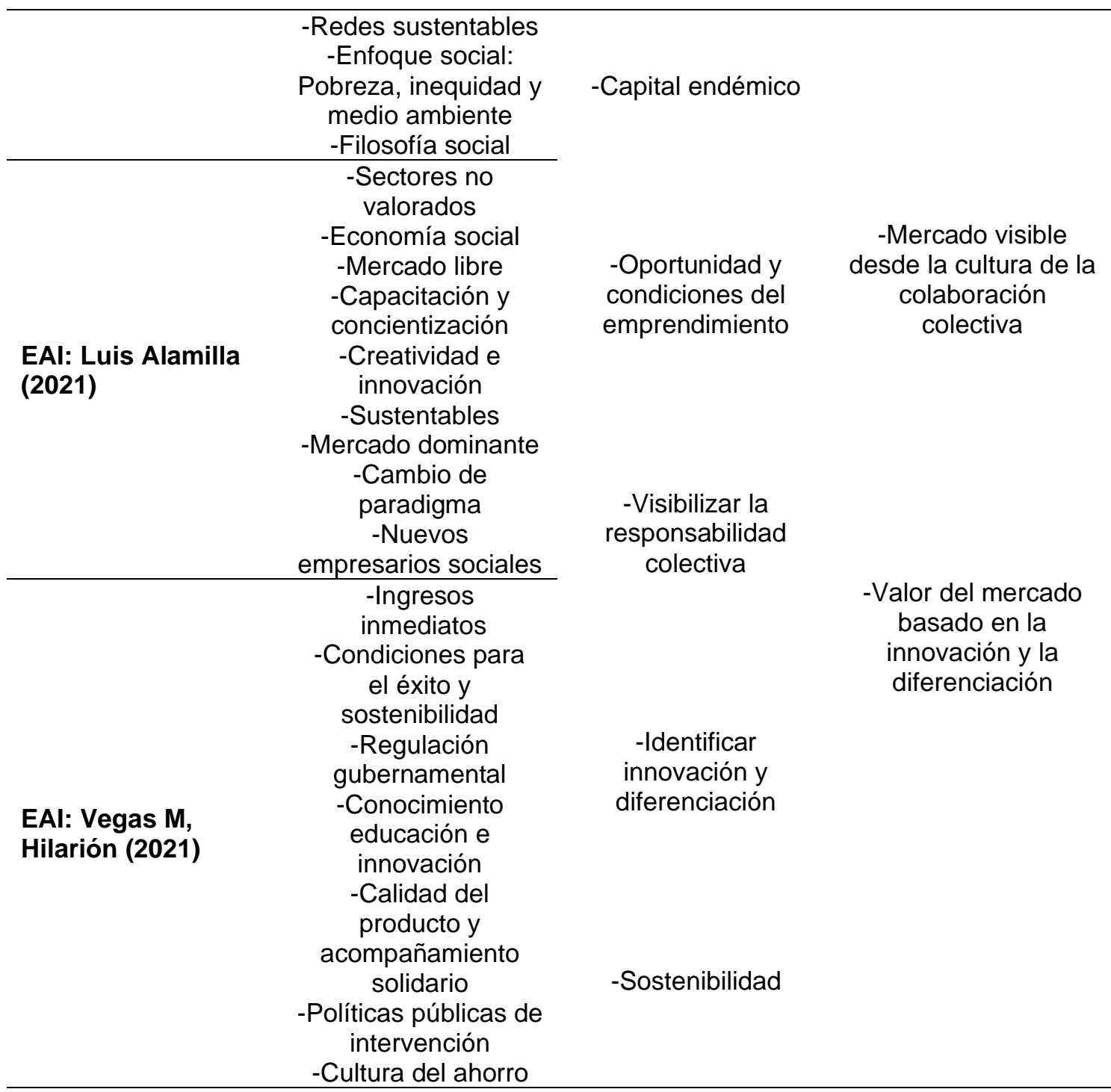

Fuente: López \& Hidalgo (2021) a partir de entrevistas en profundidad. Notas: AC: Actor de cambio; EAl: Experto Académico Investigador.

En cuanto a los descriptores emergentes, producto de la interpretación hermenéutica desarrollada por los investigadores, se pueden considerar como un aporte de la propia investigación para los estudiosos de este tipo de mercado popular y solidario. Veamos sus aspectos sustantivos:

\section{$\checkmark$ Mercado diferenciado y sustentable. El mercado}

diferenciado dentro del concepto de mercado popular y solidario puede representar, además de una oportunidad local de desarrollo cultural y artesanal, también lo pudiera representar desde el punto de vista de atractivo turístico; contribuyendo así con el desarrollo y fortalecimiento productivo local. 
Modelo autónomo de gestión. Los modelos autónomos nacen desde las bases culturales y folclóricas de las localidades, lo cual permite mayor autonomía en la expresión de los saberes artesanales, incluso ancestrales. Las iniciativas de emprendimiento en los mercados populares $y$ solidarios, en la mayor parte de las veces se inician bajo este concepto, la oportunidad de negocios es relevante en la medida que los emprendimientos estén focalizados en su propia identidad.

\section{$\checkmark$ Emprendimiento pensado en} la sostenibilidad del mercado. La sostenibilidad se basa en la posibilidad de ser perenne en el tiempo y con visión estratégica empresarial. Para ello, se hace necesario incorporar en este tipo de mercado popular y solidario variables que fortalezcan los sustentos del buen vivir y generar un capital endémico que, de respuesta a lo local, tales como redes sustentables y la colaboración social.
Mercado visible desde la cultura de la colaboración colectiva. Esta colaboración colectiva, de igual forma mencionada en el descriptor anterior, debe contribuir con la visibilidad del mercado popular y solidario en la que se resalte la representación de lo único en su espacio, resaltando la cultura y los aspectos que dan origen a las formas de convivencia de lo autóctono.

$\checkmark$ Valor del mercado basado en la innovación y la diferenciación. Los mercados adquieren su valor en la medida que son atractivos para quienes acuden a él; un mercado popular y solidario puede tener sus ventajas basadas en la innovación y la diferenciación, convirtiéndolo en una atracción más allá del precio y de la naturaleza de sus productos; sus innovaciones bien se pueden basar en la forma en que transforman sus productos y lo expresan en el mercado; su diferenciación puede sustentarse en la construcción de mercado pensado en la cultura artesanal y los saberes. 
No cabe la menor duda que esto considera la necesidad de establecer un enfoque de manera estratégica, en la que se incluyan variables como sostenibilidad, innovación y colaboración, las cuales deben ser consideradas al momento de establecer estrategias de emprendimiento en mercados de economía popular y solidaria.

\section{Conclusiones}

La investigación procede a presentar sus conclusiones en las que bien pueden sumarse otros aspectos a partir de próximas investigaciones relacionadas con los mercados populares y solidarios. Tal como se menciona en el párrafo anterior, variables como la sostenibilidad, innovación y colaboración se hacen vitales para la construcción y desarrollo de este tipo de mercados.

En relación con la sostenibilidad es importante que toda iniciativa de emprendimiento se visualice desde la perspectiva empresarial, dado que al final es un intercambio comercial sustentado sobre las bases de la confianza y por supuesto de reglas de juego que se deben de respetar; en él se debe de reforzar el tema de la inclusión, eso genera empatía y disminuye la discriminación entre los miembros de una misma comunidad; dando mayor espacio para la sensibilidad y el conocimiento de las áreas prioritarias del emprendimiento por parte del consumidor.

Desde las perspectivas de la innovación, todo producto que incluye transformaciones sustanciales y es dirigido al mercado, es innovación; lo cual por supuesto, no solo debe de ser aceptado por el consumidor, sino que a su vez muestre su utilidad; lógicamente, la innovación está asociada a la mejora de resultados, haciendo que la nueva transformación sustituya paradigmas de acercamiento al mercado, y más bien se haga desde lo autóctono.

La colaboración, inicialmente, da un matiz de compromiso entre los promotores del mercado popular y solidario; sin embargo, se hace necesario la incorporación de los "consumidores educados" para este tipo de mercado; incluso la visibilidad de la co-creación generada desde las propias bases de las comunidades frente a las 
posibilidades de generar propuestas de valor comunitario o asociativo que ayude a generar demandas futuras.

La investigación cierra una fase. Sin embargo, espera que se abran otros momentos para la disertación a partir de esta contextualización.

\section{Bibliografía}

Alamilla L. (2021). Nuevos mercados de economía popular y solidaria. Un enfoque estratégico desde el emprendimiento local. Entrevista en profundidad

Asamblea Constituyente (24 de julio de 2008). Constitución Política del Ecuador 2008. [en línea] http://biblioteca.espe.edu.ec/ upload/2008.pdf

Castro M., A. (2018). Economía popular y solidaria. ¿Realidad o utopía? Caracterización de las entidades de fomento. Universidad Politécnica Salesiana.

Cotera, A. (2008). Informe del Estudio de Caso de Perú. [En línea]

http://www.economiasolidaria .org/node/1906

Marín B., G. (2021). Nuevos mercados de economía popular y solidaria. Un enfoque estratégico desde el emprendimiento local. Entrevista en profundidad.

Organización de las Naciones Unidas (2020). Objetivos de Desarrollo Sostenible 2030. [En línea]. https://www.un.org/sustainabl edevelopment/es/objetivosde-desarrollo-sostenible/

Ramon P. (2021). Nuevos mercados de economía popular y solidaria. Un enfoque estratégico desde el emprendimiento local. Entrevista en profundidad.

Secretaria Técnica Planifica Ecuador (2020). Plan Nacional de Desarrollo 2017 - 2021 Toda una Vida. [En línea]. https://www.planificacion.gob. ec/plan-nacional-dedesarrollo-2017-2021-todauna-vida/

Strauss y Corbin (2002). Bases de la investigación cualitativa. Técnicas y procedimientos para desarrollar la teoría fundamentada. CONTUS, Editorial Universidad de Antioquia.

Unceta, K. (2014). Poscrecimiento, desmercantilización y Buen Vivir. Nueva Sociedad.

Vegas M., H. (2021). Nuevos mercados de economía popular y solidaria. Un enfoque estratégico desde el 
emprendimiento

local.

Entrevista en profundidad.

Vegas M., H. (2017). Políticas públicas y gobernanza. Articulación para una gestión pública local autónoma. Polis [en línea]. 2017, vol.16, n.48, pp.155-172. ISSN 07186568.

http://dx.doi.org/10.4067/S07 18-65682017000300155.

Vegas, H. (2016). La teoría fundamentada como herramienta metodológica para el estudio de la gestión pública local. Revista Venezolana de Gerencia, 21(75).

https://produccioncientificaluz .org/index.php/rvg/article/view /21891/21636 